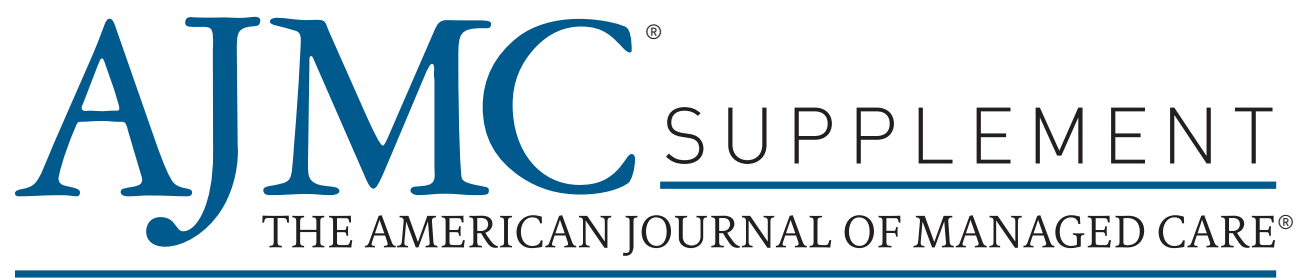

\title{
Examining Developments in Multicancer Early Detection: Highlights of New Clinical Data from Recent Conferences
}

\section{HIGHLIGHTS}

> Examining Developments in Multicancer Early Detection: Highlights of New Clinical Data from Recent Conferences

$>$ CE Posttest 


\section{Examining Developments in Multicancer Early Detection: Highlights of New Clinical Data from Recent Conferences}

Release date: December 10, 2021

Expiration date: December 10, 2022

Estimated time to complete activity: 2.0 hours

Type of activity: Application

Medium: Print with internet-based posttest, evaluation, and request for credit.

Fee: Free

This activity is supported by an educational grant from GRAIL, Inc.

\section{Intended Audience}

Managed care payers, pharmacy directors, pharmacy benefit managers, specialty pharmacy directors, and any other pharmacist and/or healthcare professional interested in the treatment of cancer.

\section{Activity Overview}

The majority of deadly cancers do not have guideline-recommended screening tests available, many cancers are detected too late, after they have progressed to late stages when chances of survival are much lower contributing to overall cancer deaths continuing to escalate worldwide. A broad set of these cancer types have been shown to be detectable simultaneously by new, noninvasive multicancer early detection (MCED) blood tests. These blood-based multicancer screening tools can potentially streamline the screening process to improve adherence and cost-effectiveness for patients. The development of new technologies for early detection of multiple types of cancer will have important clinical implications for managed care professionals involved in the identification, management, and treatment of patients with cancer. This article will review recent and ongoing advancements and present the newest clinical data surrounding multicancer detection for managed care professionals to become familiar with and potentially incorporate these preventive screening tools into treatment paradigms for patients.

\section{Statement of Need}

In the United States, cancer is the leading cause of death among those younger than 80 years of age. Due to improvements in cancer prevention, screening, treatment, and survivorship care, the mortality rates with cancer had decreased significantly in the United States between 1991 and 2015. Earlier cancer detection has been associated with improved prognosis, treatment success, and survival rates. However, just 4 routine cancer screening tests are available (breast, cervical, colorectal, and lung) and their screening rates are below national goals resulting in a large gap for cancer detection. There have been recently published data on bloodbased multicancer early detection tests that can identify multiple cancers, many for which no screening currently exists, in a single blood test. Reliably detecting cancer at earlier stages has the potential to decrease mortality and reduce overall healthcare costs by initiating treatment early. It is essential that managed care professionals are informed on recent and ongoing advances and literature with multicancer detection tests in order to make recommendations for utilization.

\section{Educational Objectives}

At the completion of this activity, the participant will be able to:

- Examine the clinical and economic burden associated with cancer, as well as the need to engage in multicancer early detection to improve overall prognosis.

- Investigate recent clinical updates for emerging and novel multicancer early detection technologies from recent conferences and publications.

- Analyze the impact of new data on managed care considerations for enhancing cancer screening and care for patients with cancer.

\section{Accreditation Statement}

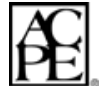
Pharmacy Times Continuing Education ${ }^{\mathrm{TM}}$ is accredited by the Accreditation Council for Pharmacy Education (ACPE) as a provider of continuing pharmacy education. This activity is approved for 2.0 contact hours $(0.20 \mathrm{CEU})$ under the ACPE universal activity number 0290-0000-21-406-H01-P. The activity is available for CE credit through December 10, 2022.

Obtaining Credit: Participants must read the article, complete the posttest and an evaluation and request for credit. Detailed instructions on obtaining CE credit are included at the end of this activity.

This CE activity is also offered free online at www.ajmc.com/ce and at www.PharmacyTimes.org/multicancer-detection, where you will be directed to the activity in its entirety, including the online pretest and posttest, activity evaluation, and request for credit. 


\section{SUPPLEMENT}

\section{Examining Developments in Multicancer Early Detection: Highlights of New Clinical Data from Recent Conferences}

\section{OVERVIEW \\ Through this article in \\ The American Journal of \\ Managed Care ${ }^{\circledR}$, managed care \\ professionals will increase \\ their knowledge regarding the \\ development of multicancer \\ early detection tests.}

\section{TABLE OF CONTENTS}

Participating Faculty

Reports

Examining Developments in Multicancer Early Detection: Highlights of New

Clinical Data from Recent Conferences

Tomasz M. Beer, MD, FACP

CE Sample Posttest

S356 


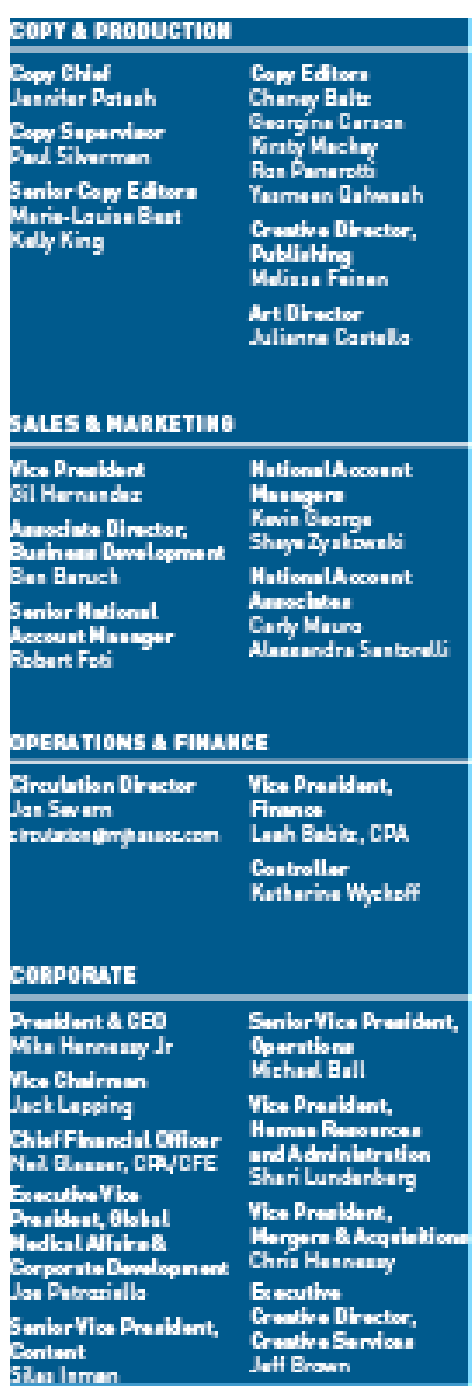
Chairman A Found der
Mike Hannaew Sr

\section{FACULTY}

Tomasz M. Beer, MD, FACP

Grover C. Bagby Endowed Chair for Prostate

Cancer Research

Deputy Director, Oregon Health \& Science

University Knight Cancer Institute

Chief Medical Officer, Center for Early Detection

Advanced Research (CEDAR)

Professor of Medicine, Hematology

\& Medical Oncology

Director, Prostate Cancer Research Program

Oregon Health \& Science University

Portland, Oregon

\section{MEDICAL WRITING \& EDITORIAL SUPPORT}

Debra Gordon, MS

President

GordonSquared, Inc

Norfolk, Virginia

\section{FACULTY DISCLOSURES}

Tomasz M. Beer, MD, FACP, has the following relevant financial relationships with commercial interests to disclose:

CONSULTANT

Arvinas, Astellas Pharma, AstraZeneca, Bayer HealthCare Pharmaceuticals, Constellation, Grail Inc., Janssen, Myovant Sciences, Pfizer, Sanofi.

\section{GRANTS/RESEARCH SUPPORT}

Paid to institution: Alliance Foundation Trials, Astellas Pharma, Bayer HealthCare Pharmaceuticals, Boehringer Ingelheim, Corcept Therapeutics, Endocyte Inc./Advanced Accelerator Applications (AAA), Freenome, Grail Inc., Harpoon Therapeutics, Janssen Research \& Development, Medivation Inc., Sotio, Theraclone Sciences/OncoResponse, Zenith Epigenetics.

STOCK/SHAREHOLDER

Arvinas, Salarius Pharmaceuticals.

\section{MEDICAL WRITING \& EDITORIAL SUPPORT DISCLOSURES}

Medical reviewer Debra Gordon, MS, has the following relevant financial relationships with commercial interests to disclose:

STOCK/SHAREHOLDER

Merck, AbbVie, and Organon.

Pharmacy Times Continuing Education ${ }^{\mathrm{TM}}$ Planning Staff: Jim Palatine, RPh, MBA; Maryjo Dixon, RPh; Karleen Melody, PharmD; Kylie Ferrentino; Susan Pordon; Brianna Winters; and Chloe Taccetta have no relevant financial relationships with commercial interests to disclose.

\section{DISCLOSURE POLICY}

According to the disclosure policy of The American Journal of Managed Care ${ }^{\circledR}$ and Pharmacy Times Continuing Education ${ }^{\mathrm{TM}}$, all persons who are in a position to control content are required to disclose any relevant financial relationships with commercial interests. If a conflict is identified, it is the responsibility of Pharmacy Times Continuing Education ${ }^{T M}$ to initiate a mechanism to resolve the conflict(s). The existence of these relationships is not viewed as implying bias or decreasing the value of the activity. All educational materials are reviewed for fair balance, scientific objectivity of studies reported, and levels of evidence.

\section{DISCLOSURE OF UNAPPROVED/OFF-LABEL USE}

The contents of this activity may include information regarding the use of products that may be inconsistent with or outside the approved labeling for these products in the United States. Participants should note that the use of these products outside current approved labeling is considered experimental and they are advised to consult prescribing information for these $\approx$ products.

The information provided in this CE activity is for continuing medical and pharmacy education purposes only and is not meant to substitute for the independent medical or pharmacy judgment of a physician or pharmacist relative to diagnostic treatment, or management options for a specific patient's medical condition.

The opinions expressed in the content are solely those of the individual faculty members and do not reflect those of The American Journal of Managed Care ${ }^{\circledR}$, Pharmacy Times Continuing Education $^{\top \mathrm{M}}$, or any of the companies that provided commercial support for this CE activity.

Signed disclosures are on file at the office of The American Journal of Managed Care ${ }^{\circledR}$, Cranbury, New Jersey. 


\title{
Examining Developments in Multicancer Early Detection: Highlights of New Clinical Data from Recent Conferences
}

\author{
Tomasz M. Beer, MD, FACP
}

\section{Introduction}

Nearly 2 million new cancers were expected to be diagnosed in the United States in 2021, with an estimated 608,570 cancer-related deaths. Despite significant advances in detection and treatment, cancer remains the second leading cause of death in the United States after cardiovascular disease (with the exception of temporal changes due to COVID-19). ${ }^{1,2}$ Delays in early diagnosis and treatment during the COVID-19 pandemic may further increase the burden of cancer in the coming years. ${ }^{1}$ Direct US medical costs for cancer in 2020 were an estimated $\$ 200.7$ billion (in 2019 dollars), an increase of $10 \%$ since $2015 .{ }^{3}$ Given the aging population, costs are expected to reach $\$ 246$ billion by 2030 .

Between 1991 and 2018, the age-adjusted cancer mortality rate in the United States declined 31\%, due, in part, to declines in smoking, improvements in early detection, and advancements in treatment for some cancers. That translates to about 3.2 million fewer cancer deaths. ${ }^{1}$ Those trends are largely driven by improvements in lung, colorectal, breast, and prostate cancer deaths. Many others (eg, pancreatic, ovarian, and liver) are primarily diagnosed in the later stages; thus, there are still disappointing 5-year survival rates. The 5-year relative survival rate (defined as the ratio of observed survival in a population to the expected or background survival rate) for pancreatic cancer, for instance, is just $10 \% .{ }^{1}$

Earlier diagnosis has the potential to reduce cancer-related deaths. One study estimated 15\% fewer cancer-related deaths if those with stage IV cancers had been diagnosed at stage III. ${ }^{4}$ Other research predicts that early cancer detection could increase 5 -year survival rates to $90 \% .^{5}$ In lung cancer, which was the leading cause of cancer death in the United States, patients diagnosed in the earliest stages have a 59\% 5-year relative survival rate, while those diagnosed in stage IV have a $6 \%$ survival rate. ${ }^{1}$ However, a study that implemented population-based ovarian cancer screening found earlier diagnosis did not reduce cancer-related deaths. ${ }^{6}$ This result is a reminder that shifts in stage at diagnosis may not always correlate with mortality reductions. Studies that measure both are important in establishing the efficacy of early detection efforts.

\section{ABSTRACT}

Cancer remains the second leading cause of death in the United States despite decades of treatment advances. While death rates have fallen for breast, cervical, and colorectal cancers, death rates remain high for the majority of malignancies, primarily given the late stage at which they are diagnosed. The US Preventive Services Task Force currently recommends routine screening for just 4 cancers: breast, cervical, colorectal, and lung (for high-risk individuals); for prostate cancer, recommendations support individual decision making. However, cancers without recommended screening tests account for $71 \%$ of cancer deaths in the United States. In addition, screening rates remain below national goals, with numerous barriers to population-based screening. Recently, initial results of studies on blood-based multicancer early detection tests, which rely on measurement of a range of analytes, demonstrate the potential to identify multiple cancers in a single blood test and detect many cancers for which no screening tests are currently recommended. Blood-based tests have the potential to be more accessible and easier to disseminate than organspecific tests. However, it remains unclear if their use can reduce deaths from these cancers. Other issues include cost-effectiveness, the impact of false-positive and false-negative results on patients and costs, and uptake among individuals and clinicians. Research and development of blood-based multicancer early detection tests continue.

Am J Manag Care. 2021;27:S347-S355

For author information and disclosures, see end of text. 


\section{Cancer Detection: Current Screening}

The US Preventive Services Task Force (USPSTF) recommends population-based screening for breast, colorectal, and cervical cancers. ${ }^{7}$ For prostate cancer, the Task Force recommends an individual decision based on a discussion of potential harms and benefits. ${ }^{8,9}$ The Task Force also recommends screening certain smokers for lung cancer. ${ }^{7}$ Table $\mathbf{1}^{7-13}$ contains a summary of cancer screening tests and recommendations from the USPSTF. Early detection as well as advances in treatment are likely behind the declining mortality rates for these cancers. However, cancers without recommended screening tests account for $71 \%$ of cancer deaths in the United States. ${ }^{10}$

The Task Force's decisions on screening recommendations are based on 4 factors ${ }^{14}$ :

1. Evidence related to benefits and harms from randomized clinical trials and observational studies.

2. Whether benefits outweigh harms and, if so, by how much and in which populations.

TABLE 1. Cancer Screening Tests and Recommendations from the USPSTF ${ }^{7-13}$

\section{Screening test} Indication USPSTF recommendation

\begin{tabular}{|c|c|}
\hline Sigmoidoscopy & $\begin{array}{l}\text { Detect abnormal } \\
\text { growth in the colon }\end{array}$ \\
\hline Colonoscopy & $\begin{array}{l}\text { Detect abnormal } \\
\text { growth in the colon }\end{array}$ \\
\hline
\end{tabular}

Mammography

Breast cancer

Human papillomavirus

Cervical cancer

Biennial screening for women aged making for women aged 40-49 years ${ }^{7}$

Every 3 years with cervical cytology alone in women aged 21-29 years; every 3 years with cervical cytology alone, every 5 years with hrHPV

Papanicolaou test

(Pap smear)

Cervical cancer

testing alone, or every 5 years with hrHPV testing in combination with cytology (co-testing) in women aged 30-65 years ${ }^{7}$

In adults aged $50-80$ years who have a 20 pack-year smoking history and Low-dose CT scan Lung cancer currently smoke or have quit within the past 15 years ${ }^{7,12}$

For men aged 55-69 years, the decision to undergo periodic PSA-based screening for prostate PSA

Prostate cancer
BRCA1/BRCA2

gene mutations

CA-125 blood test
Breast, ovarian, pancreatic cancers cancer should be an individual one and should include discussion of the potential benefits and harms of screening with their clinician 8,9

For women with a personal or family history of breast, ovarian, tubal, or peritoneal cancer ${ }^{7}$ Not recommended ${ }^{13}$
CT, computed tomography; hrHPV, high-risk human papillomavirus; PSA, prostate-specific antigen; USPSTF, US Preventive Services Task Force. financial, a lack of awareness about screening as well as provider recommendations, psychosocial and logistical barriers, a lack of trust in the healthcare system, and access, contribute to this result. ${ }^{16-19}$ Screening for cervical and breast cancer declined between 2011 and 2019 in insured populations; additionally, for cervical and colorectal cancers, screening declined in uninsured populations. ${ }^{20} \mathrm{~A}$ recent analysis of the Surveillance, Epidemiology, and End Results (SEER) Program database presented at the International Society for Pharmacoeconomics and Outcomes Research (ISPOR) 2021 virtual meeting found an increased incidence in cervical cancer among women younger than 50 years and a significant decrease in the 5-year survival rate, though the authors did not correlate it with the lower screening rate. ${ }^{21}$

Results of a study based on the 2018 Behavioral Risk Factor Surveillance System data found significant differences in meeting breast, cervical, and colorectal cancer screening guidelines among women based on age, race, and ethnicity, as well as socioeconomic and insurance status. For example, women without health insurance had a 26\% to $39 \%$ lower prevalence of breast cancer screening compared with those with insurance. Overall, women with annual household incomes less than $\$ 50,000$, education below the college level, those who live in rural counties, those without 
insurance, or those who could not pay for healthcare were less likely to be screened. ${ }^{20}$

A recent retrospective claims analysis of a large commercial and Medicare Advantage database from 2008 to February 2020 presented at Academy of Managed Care Pharmacists (AMCP) Nexus 2021 found cancer screening rates of $1.2 \%, 11.7 \%, 20.8 \%, 51.4 \%$, and $64.4 \%$ for lung, prostate, colorectal, cervical, and breast cancers, respectively, based on guidelines during that period. ${ }^{22}$

The COVID-19 pandemic exacerbated screening barriers. Early in the pandemic, medical centers closed to elective procedures, including screenings; individuals were loath to go to those still open (and even after they reopened) given fears of infection; nearly 2.7 million people lost employer-provided health insurance between April 23, 2020, and December 21, 2020, and thus coverage for screenings. ${ }^{1,23}$ In addition, several medical societies recommended postponing screening. ${ }^{24}$ One large tertiary center saw $20.6 \%$ of its mammogram appointments cancelled in March 2020 , before decreasing to $7 \%$ in August of that year. ${ }^{24}$

A retrospective cohort screening of about 60 million Medicare Advantage and commercially insured individuals found a sharp decline in screening for breast, colorectal, and prostate cancers (90.8\%, 79.3\%, and 63.4\%, respectively) between March 2020 and May 2020 compared with the same time in 2018 and 2019. Based on these declines, the authors estimated an absolute deficit in screening in the United States of 9.4 million people in 2020 (3.9 million, breast; 3.8 million, colorectal; and 1.6 million, prostate). ${ }^{25}$ This, in turn, led to delayed cancer diagnoses, which may impact survival. ${ }^{26,27}$

Another drawback to currently available screening tests is falsepositive results, which can lead to anxiety as well as unnecessary medical tests. A study presented at the ISPOR 2021 virtual meeting found that cancer screening elicits significant anxiety in patients, particularly those with a high risk of cancer, with anxiety, worry, and distress highest while waiting for the results. ${ }^{28}$ False-positive rate estimates can vary depending on what is defined as a positive result. There is evidence of false-positive rates for LDCT screening as high as $26.6 \%$; overdiagnosis of indolent cancers is also cited as a concern. ${ }^{29-31}$ In one study, the authors estimated that $49.1 \%$ of women undergoing annual mammography will have a false-positive result over 10 years, with $18.6 \%$ of those undergoing a biopsy. ${ }^{32}$ Other studies using extrapolated data estimated rates between $29 \%$ and $77 \%$ after 10 screening mammograms. ${ }^{33}$ In addition, researchers have found that $23 \%$ of those undergoing annual fecal occult blood testing over 10 years will have a false-positive result. ${ }^{34}$

Cancer screening tests also result in higher costs. One study of 1087 individuals enrolled in a large screening program with traditional screening methods for prostate, lung, colorectal, and ovarian cancer found that $43 \%$ had at least 1 false-positive result, $83 \%$ of which required follow-up care. This incurred significantly higher medical costs in the year after screening than those without
TAB LE 2. Healthy People 2030 Cancer Screening Goals ${ }^{15, a}$

\begin{tabular}{lcc} 
Cancer & $\begin{array}{c}\text { Most recent } \\
\text { participation (\%) }\end{array}$ & Target (\%) \\
\hline Breast cancer & $72.8(2018)$ & 77.1 \\
Colorectal cancer & $65.2(2018)$ & 74.4 \\
Cervical cancer & $80.5(2018)$ & 84.3 \\
Lung cancer & $2.5(2015)$ & 7.5 \\
\hline
\end{tabular}

Based on current guidelines.

false-positive results: $\$ 1024$ for women and $\$ 1175$ for men (in 2004 dollars)..$^{35}$

\section{New Paradigm of Multicancer Detection}

In recent years, several companies have started developing screening blood tests to identify numerous cancers at an early stage. Called "pan-cancer tests" or multicancer early detection (MCED) tests, they represent a novel approach to screening with the potential to identify cancers at earlier stages. ${ }^{36-38}$ These tests typically combine artificial intelligence (AI) and machine learning with assays to detect a variety of circulating analytes, often including cell-free DNA (cfDNA) for early indications of cancer (Figure $\mathbf{1}^{39}$ ). ${ }^{37,39}$

Blood-based MCED tests, which rely on measurement of a range of analytes, are being studied. These tests have several attributes that complement currently available screening tests, such as the potential to identify multiple cancers in a single blood test and detect many of the cancers for which no screening tests are currently recommended. They may also prove more accessible and easier to disseminate than more complicated, organ-specific tests. The next several paragraphs will examine the tests under investigation and available on the market.

\section{Galleri}

Galleri examines cfDNA methylation patterns across a very large number of methylation sites and uses AI analysis to recognize patterns associated with the presence of cancer as well as the likely organ of origin of the suspected malignancy. It has been shown to detect more than 50 neoplasms. ${ }^{40}$ It is currently marketed in the United States under a Clinical Laboratory Improvement Amendments (CLIA) waiver, and the manufacturer plans to submit data to the US Food \& Drug Administration in 2023. ${ }^{10}$ It is intended for use in those older than 50 years with and without additional risk factors for cancer in conjunction with currently recommended screenings. ${ }^{10,41}$

\section{The Circulating Cell-free Genome Atlas (CCGA) Study}

Galleri has been assessed in several studies. The Circulating Cell-free Genome Atlas (CCGA) is a prospective, multicenter, casecontrol, observational study conducted with specimens from 15,254 participants, $56 \%$ of whom had suspected cancer. It was divided 
FIGURE 1. Components of Liquid Biopsy ${ }^{39}$

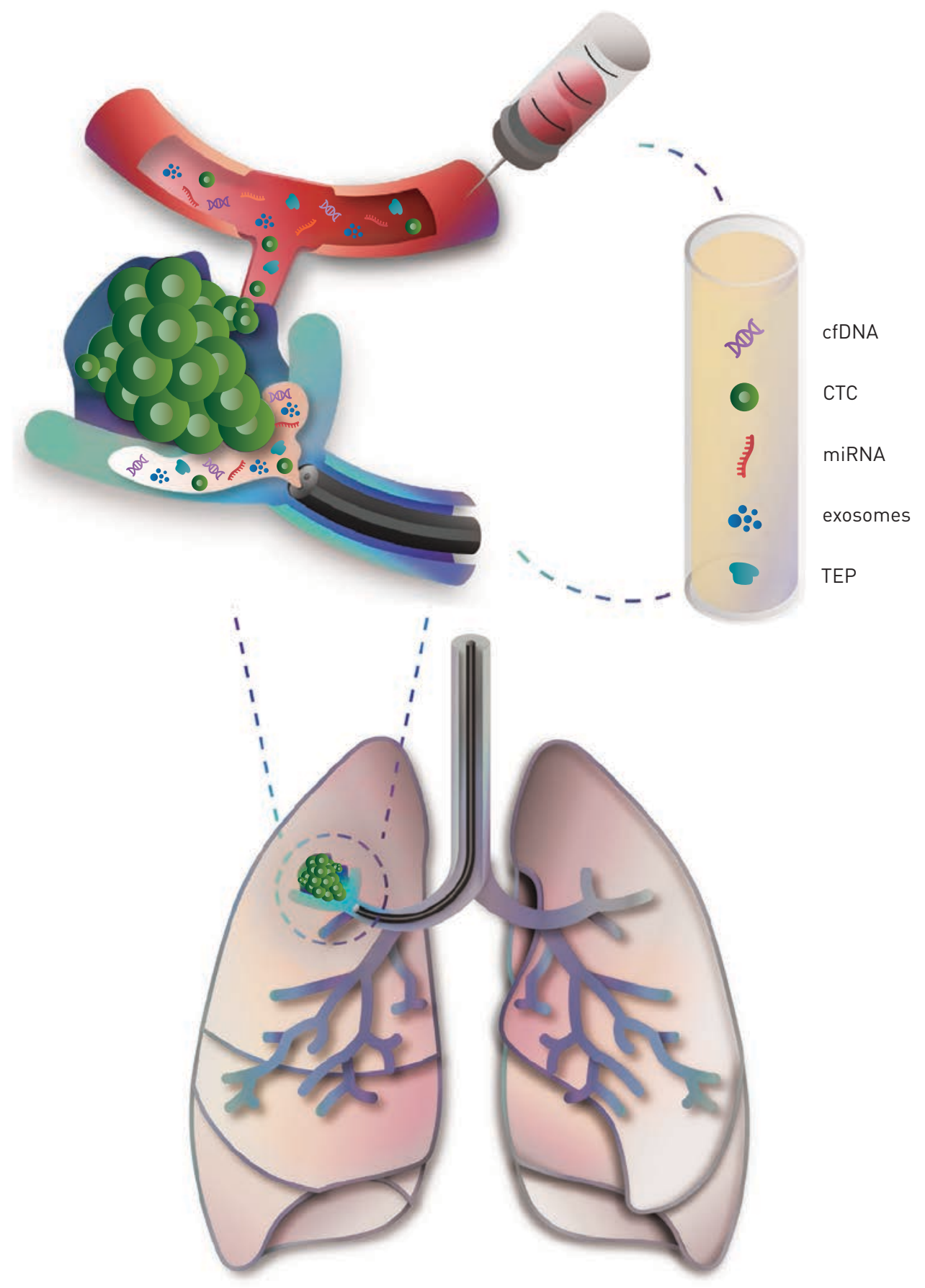

CTC, circulating tumor cells; cfDNA, circulating cell-free DNA; TEP, tumor-educated platelets.

Republished from Freitas C, Sousa C, Machado F, et al. Front Oncol. 2021;11(1130), under the terms of the Creative Commons Attribution License (CC BY). 
into 3 sub-studies: discovery, analysis (training/validation), and further validation. ${ }^{40}$

In an early sub-study, 303 participants with suspected cancer were classified as either confirmed cancer $(n=239)$ or no cancer ( $n=64)$ and evaluated for more than 20 cancer types in training and validation groups. The assay correctly predicted no cancer in the confirmed noncancer group (100\% specificity). There was a $46.7 \%$ cancer detection accuracy in the confirmed cancer validation test (35/75; $95 \%$ CI, $35.1 \%-58.6 \%$ ). The test detected $78.9 \%$ of cancers in stage II during the validation stage, with tumor of origin predicted in all validation samples (35/35) with a $97.1 \%$ accuracy. ${ }^{42}$

Results from a larger sub-study conducted in 6689 participants (2482 with cancer) found a 99.3\% specificity overall in the validation cohort for more than 50 cancers. Sensitivity for stages I-III in a prespecified set of 12 cancers that account for $63 \%$ of all cancer deaths in the United States was 67.3\% (CI, 60.7\%-73.3\%) and $43.9 \%$ (CI, 39.4\%-48.5\%) for all cancer types for early-stage detection. Figure $2^{40}$ depicts the sensitivity in individual tumors by stage,

FIGURE 2. Sensitivity in Individual Tumors by Stage ${ }^{40}$

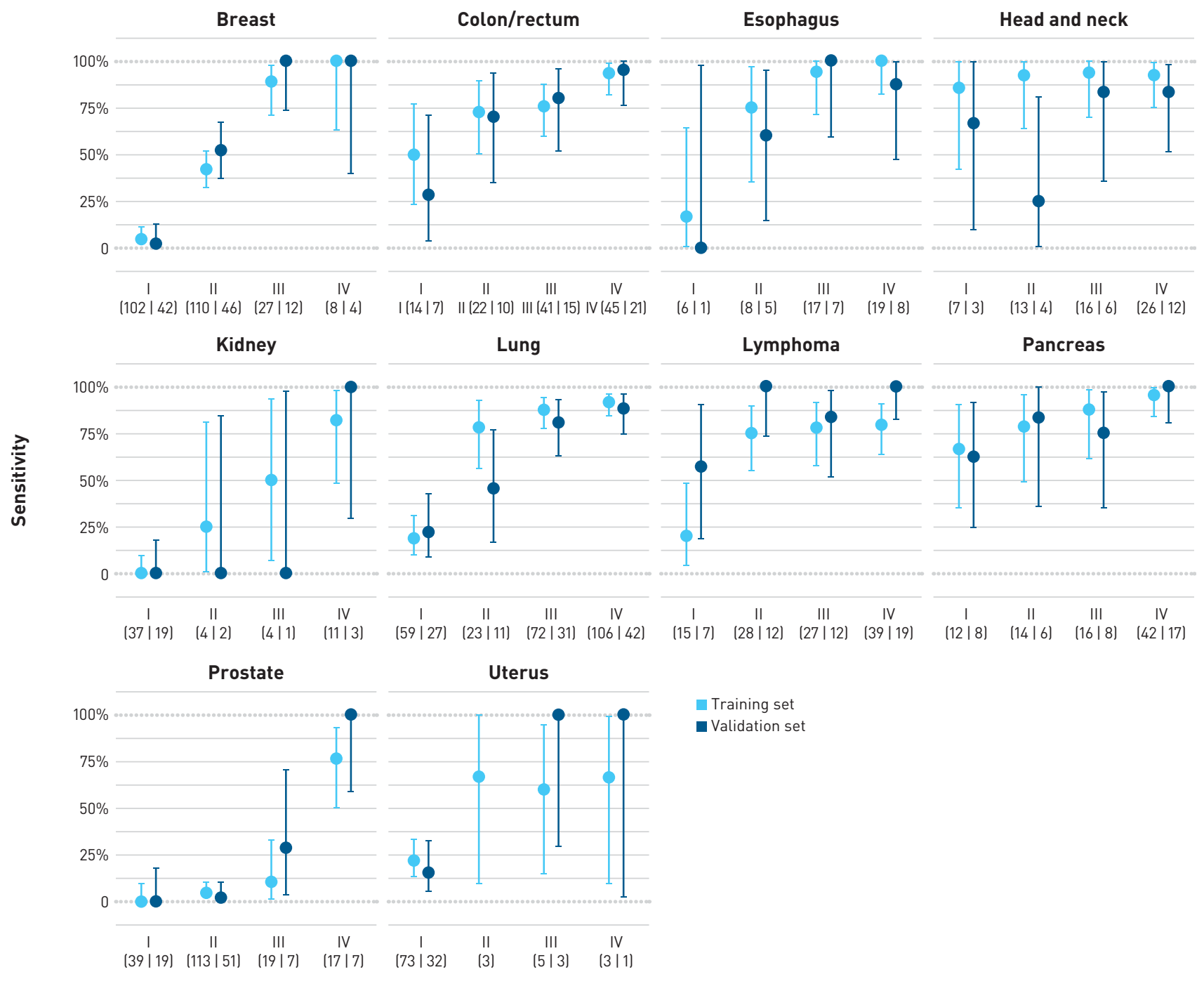

Clinical stage (n) 
with a sensitivity at $99.8 \%$ specificity (training) or $99.3 \%$ specificity (validation) with $95 \% \mathrm{CI}$ for cancers with at least 50 samples. ${ }^{40}$

A subanalysis of the potential prognostic significance of cancer detection in that study found that of those who died during a 3-year follow-up, $89 \%$ had cancer detected by the MCED test compared with $44 \%$ of those still alive at 3 years, with greater detection sensitivity among those who died. Cancers not detected by the MCED test showed better survival than expected from SEER data, while those detected by MCED test showed similar or slightly better survival. Investigators concluded that detection with the MCED test was prognostic beyond clinical stage alone $(P<.001)$ and preferentially detected more aggressive cancers. ${ }^{37}$ Participants in CCGA continue to be followed.

\section{PATHFINDER Study}

The PATHFINDER prospective study in 6629 adults 50 years and older evaluated the Galleri test's role in clinical practice. Participants and their physicians received the results of the MCED test and used those results to carry out a clinical evaluation for cancer. The clinical evaluation was not prespecified and was directed by a physician until the clinical assessment determined that diagnostic resolution had been reached. Participants and their healthcare providers received positive test results for further assessment. Interim results showed a cancer signal in 92 participants (1.4\%) with a diagnostic resolution in 65 to date. Twenty-nine of those had a true-positive and 36 had a false-positive after imaging and procedures, resulting in a positive predictive value of $45 \%$. The cancer signal origin prediction was $100 \%$ in those with first/second cancer signal origin in participants with elevated cancer risk (CI, 83.2-100) and $87.5 \%$ in those without additional cancer risk (CI, 52.9-99.4). It took about 50 days to diagnostic resolution from the time the test results were returned. The majority of cancers detected were diagnosed at stages I-III. The study is continuing. ${ }^{36}$ Additional follow-up will enable more complete assessment of test performance.

TABLE 3. DETECT-A Test Performance ${ }^{45}$

\begin{tabular}{|c|c|c|}
\hline & $\begin{array}{l}\text { Blood test without } \\
\text { confirmation }\end{array}$ & $\begin{array}{l}\text { Blood test with } \\
\text { confirmation }\end{array}$ \\
\hline Positive predictive value & $5.9 \%(4.0-8.4)$ & $19.4 \%(13.1-27.1)$ \\
\hline Specificity & $95.3 \%(94.9-95.7)$ & $98.9 \%(98.7-99.1)$ \\
\hline Negative predictive value & $99.3 \%(99.1-99.4)$ & $99.3 \%(99.1-99.4)$ \\
\hline $\begin{array}{l}\text { Number needed to screen to detect } \\
1 \text { cancer }\end{array}$ & $342(238-510)$ & $381(260-583)$ \\
\hline \multicolumn{3}{|l|}{ Sensitivity } \\
\hline All cancers & $30.2 \%(21.3-40.3)$ & $27.1 \%(18.5-37.1)$ \\
\hline Cancers with SOC screening & $27.5 \%(15.9-41.7)$ & $23.5 \%(12.8-37.5)$ \\
\hline Cancers with no SOC screening & $33.3 \%(20.0-49.0)$ & $31.1 \%(18.2-46.6)$ \\
\hline
\end{tabular}

SOC, standard of care.

\section{STRIVE Study}

The STRIVE study is an observational study to assess the ability of Galleri to detect invasive cancers in a breast cancer screening cohort of 99,308 women. Participants provide a blood sample and complete a health questionnaire at the time of screening mammogram and will be followed for up to 5 years to capture clinical information. It is currently closed to enrollment.43

\section{SUMMIT Study}

SUMMIT is a United Kingdom-based study of about 25,000 participants at high risk of lung cancer and other smoking-related cancers. Participants provide a blood sample when they have an LDCT scan. The primary outcome is to evaluate the performance of the test and examine the feasibility of delivering an LDCT screening service. ${ }^{44}$

\section{CancerSeek}

The CancerSeek test is designed to detect the presence of prespecified DNA mutation and protein markers associated with cancer. In its initial iteration, CancerSeek is designed as a 2-step test with an initial positive result triggering an additional analysis to exclude the possibility that findings reflect the presence of clonal hematopoiesis.

\section{DETECT-A Study}

The utility of this liquid biopsy test to identify cancer signals in a healthy population was evaluated in the prospective, interventional DETECT-A study. The clinical evaluation in DETECT-A was prespecified by the study design to be a positron emission tomography-computed tomography scan. Researchers screened 9911 women aged 65 to 75 years with no personal history of cancer and a high adherence to recommended screening guidelines. On the first stage of the test, $4.9 \%$ of participants scored positive, of which $1.35 \%$ (134) were confirmed in a second test component. Of those, 95\% (127) received imaging. In half, the results were suspicious for cancer and, of those 64 patients, 41\% (26) were subsequently diagnosed with cancer (Table 3$).{ }^{45}$ In addition, standard screening identified 24 cancers not found with the blood test: 20 breast cancers, 3 lung cancers, and 1 colorectal cancer. of the 24 cancers, 22 were early-stage cancers. Forty-six additional cancers were diagnosed clinically with neither the CancerSeek test nor standard screening tests during the course of the study. ${ }^{45}$

\section{DELFI}

The DELFI test is based on fragmentomes, disordered DNA packaging in cancer cells, and uses machine learning algorithms to assess patterns. It was evaluated in the LUCAS trial, 
which enrolled 365 individuals at high risk for lung cancer and with smoking-related symptoms. In this pilot study, the DELFI approach performed well. The receiver operator curve, which integrates sensitivity and specificity for cancer detection, showed an area under the curve of 0.9. ${ }^{38}$

An earlier study analyzed fragmentation profiles of 236 patients with breast, colorectal, lung, ovarian, pancreatic, gastric, or bile duct cancer and 245 healthy individuals. It found a sensitivity ranging from $57 \%$ to $99 \%$ among the 7 cancers with a $98 \%$ specificity. The tissue of origin was identified in $75 \%$ of cases. Overall, it detected cancer in $91 \%$ of the patients with cancer. ${ }^{46}$

Several trials are also underway to use AI and blood-based screening for colorectal cancer. ${ }^{47-49}$ Early results presented at the 2020 Digestive Disease Week suggest high specificity and sensitivity. ${ }^{50}$

\section{Managed Care Concerns}

The economic burden associated with cancer is related to the stage at which the cancer is diagnosed. An analysis of a SEER-Medicare database found the highest costs in the most advanced stage of cancer, or the end-of-life stage, ranging from $\$ 71,000$ for prostate cancer to $\$ 239,000$ for acute myeloid leukemia. Costs were $\$ 41.8$ billion for all cancer sites treated in the early stage and $\$ 105.5$ billion for patients in the end-of-life stage. ${ }^{51}$

A more recent study presented at AMCP Nexus 2021 among 35,817 patients with cancer between 2016 and 2020 found absolute mean cost in stage I ovarian cancer of $\$ 36,000$ but that cost increased to $\$ 169,999$ by stage IV, a more than 4 -fold difference. Costs for colorectal cancer were nearly doubled, from $\$ 82,000$ to $\$ 157,999 .{ }^{52}$ Thus, earlier diagnosis could have economic as well as lifesaving benefits provided that it is not associated with a high burden of overdiagnosis and overtreatment. . $^{33,54}$

However, the costs of testing would also have to be considered. MCED tests are largely still investigational with just one, Galleri, currently marketed in the United States at a cost of $\$ 949$ per test. ${ }^{55}$ Up to 100 million Americans could be eligible for this test, which is being developed for use in individuals 50 years and older. The cost, if $50 \%$ of potentially eligible Americans were tested, would approach $\$ 50$ billion, with additional costs for the testing and treatment that would follow in those with identifiable cancers. The frequency with which such testing might be repeated has not been defined yet, so it is not possible to estimate annual costs. Economic analyses of these tests are just beginning to emerge.

A Markov model comparing annual MCED test plus standard of care (SOC) screening in US adults aged 50 to 79 years predicted that $10.7 \%$ of cancers would be detected at stage IV by adding an MCED test compared with $20.4 \%$ with SOC screening. That led to 0.17 more quality-adjusted life-years (QALYs) and reduced cancer-related treatment and diagnosis costs by $\$ 5208$. At a willingness-to-pay (WTP) threshold of $\$ 50,000 / \mathrm{QALY}$, the value-based price would be
\$923 per test; at a WTP threshold of \$100,000/QALY, the value-based price would be $\$ 2069$ per test. ${ }^{56}$ These estimates represent a set of assumptions that will need to be confirmed as testing becomes more widely available.

One model compared colorectal screening methods (capsule endoscopy every 5 or 10 years; computed tomography colonography [CTC] every 5 years; multitarget stool DNA every 1 or 3 years; or the methylated mSEPT9 DNA plasma assay every 1 or 2 years against annual fecal immunochemical testing [FIT] screening or colonoscopy every 10 years). At a WTP threshold of $\$ 100,000$ and the assumption of perfect adherence, the authors found that an annual mSEPT9 test resulted in more QALYs gained and colorectal cases and deaths avoided than annual FIT screening, but high rates of colonoscopy referral (51\% after 3 years; $69 \%$ after 5 years). In addition, a CTC every 5 years and annual screening with the mSEPT9 every year were efficient strategies, with incremental cost-effectiveness ratios (ICERs) of $\$ 1092$ and $\$ 63,253$, respectively. ${ }^{57}$

A recent health economic model comparing the clinical outcomes of adding an MCED test to current cancer screening versus current screening alone over a lifetime in people aged 50 to 79 years found those receiving an MCED test could have an incremental gain of 0.35 life-years and 0.34 QALYs per person, primarily due to earlier detection of cancers and improved mortality. At WTP thresholds of $\$ 100,000$ to $\$ 150,000 / Q A L Y$, that translated to a cost benefit of $\$ 34,400$ to $\$ 51,600$ per person. ${ }^{58}$

A study estimating the impact of adding MCED screening for breast, cervical, colorectal, and lung cancers to current screening modalities estimated it would detect an additional 105,526 of these and other cancers with a 25\% uptake and 422,105 with a $100 \%$ uptake. The estimated true-positive/false-positive (TP/FP) ratio, which demonstrates how many patients without cancer should undergo diagnostic evaluation to detect 1 person with cancer, was $1: 43$ ( $\$ 89,042$ per cancer detected) with the 4 SOC tests and 1:1.8 with MCED ( $\$ 7060$ per cancer detected). Combining the two yielded a TP/FP ratio of 1:14 in the United States, with diagnostic costs per cancer of $\$ 32,461 . .^{59}$

The authors noted that while reported sensitivities for MCED tests tended to be lower than currently used screening tests, they cover multiple cancers and have a relatively low false-positive rate, thus their impact in screening is expected to be greater. They also noted that a single test could overcome the high rates of nonadherence seen in the United States. ${ }^{59}$ Naturally, all of these economic analyses are preliminary and built on a set of assumptions that will need to be further confirmed within clinical trials and real-world evidence-gathering efforts.

Given that just 1 MCED test has been marketed for 5 months at the time of this writing, uptake is unclear. However, an online discrete choice experiment answered by 303 individuals found that fewer false-negative and more true-positive rates were the most 
important considerations for an MCED screening test. In addition, $71.9 \%$ reported a preference for the MCED test with currently recommended screenings. ${ }^{60}$ Another consideration is that the lower false-positive rates of MCEDs could reduce patient anxiety as well as costs. ${ }^{35,61}$

\section{Conclusions}

Early diagnosis has the potential to improve cancer survival. Yet the majority of cancers are diagnosed in the late stages given a lack of effective population-based screening tests. Cancers without recommended screening tests account for $71 \%$ of cancer deaths in the United States. ${ }^{8}$ Current recommended screening tests reduce cancer mortality. However, they experience significant falsepositive rates, invoke anxiety in patients, and have relatively less than optimal adherence rates. But, recently, several MCEDs have demonstrated the ability to detect cancers in healthy people and those with the disease. Preliminary estimates suggest promising false-positive rates and economic analyses suggest that such tests may prove to be cost-effective. ${ }^{36-38}$ Further evidence that will inform understanding of test performance is expected. Currently, just 1 MCED test is on the market under a CLIA exception, and none have been approved by the FDA for marketing. The USPSTF has not made any recommendation on their use for population screening. As with any screening tests, the MCED tests must be carefully validated in prospective, population-based, long-term studies to determine not only their accuracy at detecting cancer but also their clinical utility to improve cancer outcomes. The economic costs of such tests, including direct testing costs as well as diagnostic evaluation for false-positive results, must be considered as should the emotional costs for patients who receive false-positive results. Widespread implementation will require additional evidence as well as extensive education of both providers and the public. Further economic analyses will inform payers' reimbursement decisions. $\square$

Author affiliation: Tomasz M. Beer, MD, FACP, is Deputy Director of Oregon Health \& Science University Knight Cancer Institute; Chief Medical Officer, Center for Early Detection Advanced Research; Director, Prostate Cancer Research Program; and Professor of Medicine, Hematology \& Medical Oncology, Oregon Health \& Science University, Portland, OR.

Funding source: This activity is supported by an educational grant from GRAIL, Inc.

Author disclosure: Dr Beer has the following relevant financial relationships with commercial interests to disclose:

CONSULTANT: Arvinas, Astellas Pharma, AstraZeneca, Bayer HealthCare Pharmaceuticals, Constellation, Grail Inc., Janssen, Myovant Sciences, Pfizer, Sanofi.

GRANTS/RESEARCH SUPPORT: Paid to institution; Alliance Foundation Trials, Astellas Pharma, Bayer HealthCare Pharmaceuticals, Boehringer Ingelheim, Corcept Therapeutics, Endocyte Inc./Advanced Accelerator Applications (AAA), Freenome, Grail Inc., Harpoon Therapeutics, Janssen Research \& Development, Medivation Inc., Sotio, Theraclone Sciences/ OncoResponse, Zenith Epigenetics.

STOCK/SHAREHOLDER: Arvinas, Salarius Pharmaceuticals.
Author information: Substantial contributions to analysis and interpretation of data, critical revision of the manuscript for important intellectual content, and supervision.

Address correspondence to: beert@ohsu.edu

Medical writing and editorial support provided by: Debra Gordon, MS

\section{REFERENCES}

1. American Cancer Society. Cancer Facts \& Figures 2021. American Cancer Society; 2021. Accessed November 11, 2021. cancer.org/content/dam/cancer-org/research/cancer-facts-and-statistics/annualcancer-facts-and-figures/2021/cancer-facts-and-figures-2021.pdf

2. Ortaliza J, Orgera K, Kruticka A, Cox C. COVID-19 continues to be a leading cause of death in the U.S. in September 2021. Kaiser Family Foundation. Published October 13, 2021. Accessed November 11, 2021. $\mathrm{kff} .0 \mathrm{rg} / \mathrm{coronavirus}$-covid-19/issue-brief/covid-19-continues-to-be-a-leading-cause-of-death-in-the-u-s/ 3. Mariotto AB, Enewold L, Zhao J, Zeruto CA, Yabroff KR. Medical care costs associated with cancer survivorship in the United States. Cancer Epidemiol Biomarkers Prev. 2020;29(7):1304-1312. doi: 10.1158/1055-9965.EPI-19-1534

4. Clarke CA, Hubbell E, Kurian AW, Colditz GA, Hartman A-R, Gomez SL. Projected reductions in absolute cancer-related deaths from diagnosing cancers before metastasis, 2006-2015. Cancer Epidemiol Biomarkers Prev. 2020;29(5):895-902. doi: 10.1158/1055-9965.EPI-19-1366

5. Eftimie R. Hassanein E. Improving cancer detection through combinations of cancer and immune biomarkers: a modelling approach. J Transl Med. 2018;16(1):73. doi: 10.1186/s12967-018-1432-8 6. Menon U, Gentry-Maharaj A, Burnell M, et al. Ovarian cancer population screening and mortality after long-term follow-up in the UK Collaborative Trial of Ovarian Cancer Screening (UKCTOCS): a randomised controlled trial. Lancet. 2021;397(10290):2182-2193. doi: 10.1016/S0140-6736(21)00731-5

7. United States Preventive Services Task Force. A and B recommendations. Published 2021. Accessed October 3, 2021. uspreventiveservicestaskforce.org/uspstf/recommendation-topics/uspstf-and-brecommendations

8. Grossman DC, Curry SJ, Owens DK, et al; US Preventive Services Task Force. Screening for prostate cancer: US Preventive Services Task Force recommendation statement. JAMA. 2018;319(18):1901-1913. doi: 10.1001/jama.2018.3710

9. US Preventive Services Task Force. Prostate cancer screening. Published May 8, 2018. Accessed August 3, 2021. uspreventiveservicestaskforce.org/uspstf/recommendation/prostate-cancer-screening 10. Securities and Exchange Commission. Form S-1 Registration Statement: Grail, Inc.

Published September 9, 2020. Accessed October 18, 2021. sec.gov/Archives/edgar/ data/1699031/000162827920000227/grails-1.htm

11. Davidson KW, Barry MJ, Mangione CM, et al; US Preventive Services Task Force. Screening for colorectal cancer: US Preventive Services Task Force recommendation statement [published correction appears in JAMA. 2021;326(8):773]. JAMA. 2021;325(19):1965-1977. doi: 10.1001/jama.2021.6238 12. Krist AH, Davidson KW, Mangione CM, et al; US Preventive Services Task Force. Screening for lung cancer: US Preventive Services Task Force Recommendation Statement. JAMA. 2021;325(10):962-970. doi: 10.1001/jama.2021.1117

13. Grossman DC, Curry SJ, Owens DK, et al; US Preventive Services Task Force. Screening for ovarian cancer: US Preventive Services Task Force Recommendation Statement. JAMA. 2018;319(6):588-594. doi: 10.1001/jama.2017.21926

14. US Preventive Services Task Force. Use of decision models in the development of evidencebased clinical preventive services recommendations. Published May 2019. Accessed August 9, 2021. uspreventiveservicestaskforce.org/uspstf/about-uspstf/methods-and-processes/use-decision-modelsdevelopment-evidence-based-clinical-preventive-services-recommendations

15. Office of Disease Prevention and Health Promotion. Healthy People 2030: cancer. Accessed October 19, 2021. health.gov/healthypeople/objectives-and-data/browse-objectives/cancer

16. Castro S, Sosa E, Lozano V, et al. The impact of income and education on lung cancer screening utilization, eligibility, and outcomes: a narrative review of socioeconomic disparities in lung cancer screening. J Thorac Dis. 2021;13(6):3745-3757. doi: 10.21037/jtd-20-3281

17. Moss JL, Leach K, Stoltzfus KC, et al. Multilevel associations with cancer screening among women in rural, segregated communities within the northeastern USA: a mixed-methods study. J Cancer Educ. 2021:1-11. doi: 10.1007/s13187-021-02069-0

18. Moss JL, Popalis M, Ramirez SI, Reedy-Cooper A, Ruffin MT. Disparities in cancer screening: the role of county-level metropolitan status and racial residential segregation. J Community Health. Published online September 22, 2021. doi: 10.1007/s10900-021-01035-7

19. Zhu X, Parks PD, Weiser E, Jacobson DJ, Limburg PJ, Finney Rutten LJ. Barriers to utilization of three colorectal cancer screening options - data from a national survey. Prev Med Rep. 2021;24:101508. doi: 10.1016/j.pmedr.2021.101508

20. Benavidez GA, Zgodic A, Zahnd WE, Eberth JM. Disparities in meeting USPSTF breast, cervical, and colorectal cancer screening guidelines among women in the United States. Prev Chronic Dis. 2021;18:E37. doi: $10.5888 / p c d 18.200315$

21. Chaudhuri M, Hait A, Das S, Kathe N. Incidence and long-term survival of cervical cancer patients in the United States: an analysis of SEER Database. Paper presented at ISPOR 2021 Virtual. ispor.org/heorresources/presentations-database/presentation/intl2021-3339/110955

22. Kim A, Gitlin M. Fadli E, McGarvey N, Cong Z, Chung K. Adherence to breast, colorectal, lung, prostate, and cervical cancer screening guidelines in a large commercial and Medicare Advantage Plan. Paper presented at AMCP Nexus 2021 Virtual. jmcp.org/doi/pdf/10.18553/jmcp.2021.27.issue-10-a 23. Bundorf MK, Gupta S, Kim C. Trends in US health insurance coverage during the COVID-19 pandemic. JAMA Health Forum. 2021;2(9):e212487-e212487. doi: 10.1001/jamahealthforum.2021.2487

24. Pairawan SS, Olmedo Temich L, de Armas S, et al. Recovery of screening mammogram cancellations during COVID-19. Am Surg. Published online October 10, 2021. doi: 10.1177/00031348211051695 
25. Chen RC, Haynes K, Du S, Barron J, Katz AJ. Association of cancer screening deficit in the United States with the COVID-19 pandemic. JAMA Oncology. 2021;7(6):878-884. doi: 10.1001/ jamaoncol.2021.0884

26. Knoll K, Reiser E, Leitner K, et al. The impact of COVID-19 pandemic on the rate of newly diagnosed gynecological and breast cancers: a tertiary center perspective. Arch Gynecol Obstet. Published online September 24, 2021. doi: 10.1007/s00404-021-06259-5

27. Xhou J, Ramsey C, Akhondzadeh MA, et al. The impact of the COVID-19 pandemic on stage at diagnosis of breast and colorectal cancers. Paper presented at ASCO 2021 Virtual. J Clin Oncol. 2021;39(15 suppll:6501. doi: 10.1200/JC0.2021.39.15 suppl.6501

28. Kim A, Chung KC, Keir C, Patrick D. Patient-reported outcomes associated with cancer screening: a systematic review. Paper presented at ISPOR 2021 Virtual. ispor.org/heor-resources/presentationsdatabase/presentation/intl2021-3339/110551

29. Pinsky PF, Gierada DS, Black W, et al. Performance of Lung-RADS in the National Lung Screening Trial: a retrospective assessment. Ann Intern Med. 2015;162(7):485-491. doi: 10.7326/M14-2086 30. Pinsky PF, Bellinger CR, Miller DP, Jr. False-positive screens and lung cancer risk in the National Lung Screening Trial: implications for shared decision-making. J Med Screen. 2018:25(2):110-112. doi: $10.1177 / 0969141317727771$

31. Thalanayar PM, Altintas N, Weissfeld JL, Fuhrman CR, Wilson DO. Indolent, potentially inconsequential lung cancers in the Pittsburgh Lung Screening Study. Ann Am Thorac Soc. 2015;12(8):1193-1196. doi: 10.1513/AnnalsATS.201412-5770C

32. Elmore JG, Barton MB, Moceri VM, Polk S, Arena PJ, Fletcher SW. Ten-year risk of false positive screening mammograms and clinical breast examinations. N Engl J Med. 1998;338(16):1089-1096. doi: 10.1056/NEJM199804163381601

33. Hubbard RA, Kerlikowske K, Flowers Cl, Yankaskas BC, Zhu W, Miglioretti DL. Cumulative probability of false-positive recall or biopsy recommendation after 10 years of screening mammography: a cohort study. Ann Intern Med. 2011;155(8):481-492. doi: 10.7326/0003-4819-155-8-201110180-00004 34. Hubbard RA, Johnson E, Hsia R, Rutter CM. The cumulative risk of false-positive fecal occult blood test after 10 years of colorectal cancer screening. Cancer Epidemiol Biomarkers Prev. 2013;22(9):16121619. doi: 10.1158/1055-9965.EPI-13-0254

35. Lafata JE, Simpkins J, Lamerato L, Poisson L, Divine G, Johnson CC. The economic impact of falsepositive cancer screens. Cancer Epidemiol Biomarkers Prev. 2004:13(12):2126-2132.

36. Beer TM, McDonnell CH, Nadauld L, et al. Interim results of PATHFINDER, a clinical use study using a methylation-based multi-cancer early detection test. Paper presented at American Society of Clinical Oncologists Virtual. June 2021. J Clin Oncol. 2021;39(15 suppl):3010. doi: 10.1200/JC0.2021.39.15_ suppl.3010

37. Chen X, Dong Z, Hubbell E, et al. Prognostic significance of blood-based multi-cancer detection in plasma cell-free DNA. Clin Cancer Res. 2021-27(15):4221-4229. doi: 10.1158/1078-0432.CCR-21-0417

38. Mathios D, Johansen JS, Cristiano S, et al. Detection and characterization of lung cancer using cellfree DNA fragmentomes. Nat Commun. 2021;12(1):5060. doi: 10.1038/s41467-021-24994-w

39. Freitas C, Sousa C, Machado F, et al. The role of liquid biopsy in early diagnosis of lung cancer. Front Oncol. 2021;11:634316. doi: 10.3389/fonc.2021.634316

40. Liu MC, Oxnard GR, Klein EA, Swanton C, Seiden MV; CCGA Consortium. Sensitive and specific multi-cancer detection and localization using methylation signatures in cell-free DNA. Ann Oncol. 2020;31(6):745-759. doi: 10.1016/j.annonc.2020.02.011

41. Cairns E. Grail launches pan-cancer screen - for those who can pay out of pocket. Evaluate Vantage. Published June 4, 2021. Accessed October 3, 2021. evaluate.com/vantage/articles/news/snippets/graillaunches-pan-cancer-screen-those-who-can-pay-out-pocket
42. Thiel D, Chen X, Kurtzman KN, et al. Prediction of cancer and tissue of origin in individuals with suspicion of cancer using a cell-free DNA multi-cancer early detection test. Paper presented at American Association for Cancer Research 2020 Virtual. Abstract CTO21. cancerres.aacrjournals.org/content/80/16_Supplement/CTO21 43. The STRIVE Study: Development of a Blood Test for Early Detection of Multiple Cancer Types. ClinicalTrials.gov. ClinicalTrials.gov identifier: NCT03085888. Updated July 31, 2020. Accessed October 6, 2021. clinicaltrials.gov/ct2/show/NCT03085888

44. Grail. SUMMIT Study. Accessed October 6, 2021. grail.com/clinical-studies/summit-study/ 45. Lennon AM, Buchanan AH, Kinde I, et al. Feasibility of blood testing combined with PET-CT to screen for cancer and guide intervention. Science. 2020;369(6499):eabb9601. doi: 10.1126/science.abb9601 46. Cristiano S, Leal A, Phallen J, et al. Genome-wide cell-free DNA fragmentation in patients with cancer. Nature. 2019;570(7761):385-389. doi: 10.1038/s41586-019-1272-6

47. Evaluation of the ctDNA LUNAR Test in an Average Patient Screening Episode (ECLIPSE). ClinicalTrials. gov. ClinicalTrials.gov identifier: NCT04136002. Published 2021. Accessed August 5, 2021. clinicaltrials. gov/ct2/show/NCTO4136002

48. Al-EMERGE: Development and Validation of a Multi-analyte, Blood-based Colorectal Cancer Screening Test. ClinicalTrials.gov. ClinicalTrials.gov identifier: NCT03688906. Updated May 20, 2020. Accessed October 5, 2021. clinicaltrials.gov/ct2/show/NCT03688906

49. Prevention of Colorectal Cancer Through Multiomics Blood Testing (PREEMPT CRC). ClinicalTrials.gov. ClinicalTrials.gov identifier: NCT04369053. Updated August 5, 2021. Accessed October 5, 2021. clinicaltrials.gov/ct2/show/NCT04369053

50. Dean J, et al. Plasma based cell-free circulating tumor DNA (ctDNA) assessment for non-invasive detection of colorectal cancer (CRC). Paper presented at Digestive Disease Week 2020 Virtual. 51. Mariotto AB, Yabroff KR, Shao Y, Feuer EJ, Brown ML. Projections of the cost of cancer care in the United States: 2010-2020. J Natl Cancer Inst. 2011;103(2):117-128. doi: 10.1093/jnci/dja495 52. McGarvey N, Gitlin M, Qi J, Chung K. Increasing healthcare costs by stage and over time among patients diagnosed with cancer: 2008-2020. Paper presented at AMCP Nexus 2021 Virtual. jmcp.org/doi/ pdf/10.18553/jmcp.2021.27.issue-10-a

53. Kakushadze Z, Raghubanshi R, Yu W. Estimating cost savings from early cancer diagnosis. Data. 2017;2(30):2-16. doi: 10.3390.data2030030

54. World Health Organization. Guide to Cancer Early Diagnosis. World Health Organization; 2017. Accessed October 3, 2021. apps.who.int/iris/handle/10665/254500

55. Welch HG. Liquid biopsy: misplaced faith in early cancer detection? STAT. Published August 31, 2021 Accessed October 18, 2021. statnews.com/2021/08/31/liquid-biopsy-misplaced-faith-in-early-cancer-detection/ 56. Tafazzoli A, Ramsey SD, Shaul A, et al. Drivers of value-based price (VBP) for a multi-cancer early detection (MCED) test. Value Health. 2021;24(12):S2.

57. Peterse EFP, Meester RGS, de Jonge L, et al. Comparing the cost-effectiveness of innovative colorectal cancer screening tests. J Natl Cancer Inst. 2021;113(2):154-161. doi: 10.1093/jnci/djaa103 58. Ortendahl J, Lee J, Hubbell E, Clarke CA, Chung KC, Kansal AR. Projected lifetime clinical value of a multicancer early detection test. Paper presented at ISPOR 2020 Virtual. ispor.org/heor-resources/ presentations-database/presentation/intl2020-3182/102294

59. Hackshaw A, Cohen SS, Reichert H, Kansal AR, Chung KC, Ofman JJ. Estimating the population health impact of a multi-cancer early detection genomic blood test to complement existing screening in the US and UK. Br J Cancer. 2021;125(10):1432-1442. doi: 10.1038/s41416-021-01498-4 60. Gelhorn H, Ross M, Kansal AR, et al. Patient preferences for attributes of a multi-cancer early detection test: a Discrete Choice Experiment (DCE) Quantitative Pilot Study. Value Health. 2021;24(12):S2. 61. Braunstein GD, Ofman JJ. Criteria for evaluating multi-cancer early detection tests. touchReviews Oncol Haematol. 2021;17(1):3-6. doi: 10.17925/0HR.2021.17.1.3 


\section{Examining Developments in Multicancer Early Detection: Highlights of New Clinical Data from Recent Conferences}

Release date: December 10, 2021

Expiration date: December 10, 2022

Pharmacy Credit

Instructions for Receiving Continuing Pharmacy Education (CPE) Credit: Testing Information

This lesson is free online; request your CE credit at

www.PharmacyTimes.org.
Sample of Online Posttest

Choose the best answer for each of the following:

1. By 2030, direct costs for cancer in the United States are expected to reach:
A. $\$ 186$ billion
B. $\$ 210$ billion
C. $\$ 246$ billion
D. $\$ 304$ billion

2. Diagnosing stage IV cancers just 1 stage earlier could lead to how many fewer cancer-related deaths in the United States?
A. $5 \%$
B. $15 \%$
C. $20 \%$
D. $23 \%$

3. JK is a 45 -year-old man with a 20 pack-year smoking history. Which of the following screenings would be recommended for JK under current guidelines?
A. He should undergo low-dose computed tomography screening.
B. He should have a chest $x$-ray.
C. He should have a liquid biopsy.
D. Current guidelines do not recommend any screening at his age.

4. How many pan-cancer tests has the FDA approved?
A. None
B. 1
C. 2
D. 3

5. In the early Circulating Cell-free Genome Atlas (CCGA) sub-study with 303 participants, what was the specificity in the noncancer participants?
A. $80 \%$
B. $87 \%$
C. $99.3 \%$
D. $100 \%$

\section{Testing Directions}

1. Each participant evaluating the activity is eligible to receive CE credit.

2. To receive your credit online, go to www.PharmacyTimes.org and complete the online posttest and the online activity evaluation form before the expiration date. Your CE credit will be automatically uploaded to CPE Monitor. Please ensure that your Pharmacy Times ${ }^{\circledR}$ account is updated with your NABP e-profile ID number and your date of birth (MMDD format). Participation data will not be uploaded into CPE Monitor if you do not have your NABP e-profile ID number and date of birth entered into your profile on www.PharmacyTimes.org.

6. In the larger CCGA subanalysis of 6689 participants with or without cancer, what was the overall specificity rate for more than $\mathbf{5 0}$ cancers?
A. $80 \%$
B. $87 \%$
C. $99.3 \%$
D. $100 \%$

7. What was the goal of the PATHFINDER study?

A. To evaluate the test's role in clinical practice.

B. To provide prognostic data on cancer deaths cased on test results.

C. To validate the safety of the test.

D. To determine the false-positive rate.

8. Which study is testing women when they go in for their screening mammogram?
A. SUMMIT
B. STRIVE
C. DETECT-A
D. DELFI

9. The highest costs for cancer occur in which stage?
A. I
B. II
C. III
D. IV

10. Which colorectal screening method was found to be the most cost-effective at a willingness-to-pay threshold of $\$ 100,000$ and assuming perfect adherence?
A. Galleri
B. Colonoscopy
C. FIT
D. MSEPT9 


\title{
SUPPLEMENT POLICY STATEMENT
}

\author{
Standards for Supplements to The American Journal of Managed Care ${ }^{\circledR}$
}

All supplements to The American Journal of Managed Care ${ }^{\circledR}$ are designed to facilitate and enhance ongoing medical education in various therapeutic disciplines. All Journal supplements adhere to standards of fairness and objectivity, as outlined below. Supplements to The American Journal of Managed Care ${ }^{\circledR}$ will:

I. Be reviewed by at least 1 independent expert from a recognized academic medical institution.

II. Disclose the source of funding in at least 1 prominent place.

III. Disclose any existence of financial interests of supplement contributors to the funding organization.

IV. Use generic drug names only, except as needed to differentiate between therapies of similar class and indication.

V. Be up-to-date, reflecting the current las of date of publication) standard of care.

VI. Be visually distinct from The American Journal of Managed Care ${ }^{\circledR}$.

VII. Publish information that is substantially different in form and content from that of the accompanying edition of The American Journal of Managed Care ${ }^{\circledR}$.

VIII. Prohibit excessive remuneration for contributors and reviewers.

IX. Carry no advertising.

Publisher's Note: The opinions expressed in this supplement are those of the authors, presenters, and/or panelists and are not attributable to the sponsor or the publisher, editor, or editorial board of The American Journal of Managed Care ${ }^{\circledR}$. Clinical judgment must guide each professional in weighing the benefits of treatment against the risk of toxicity. Dosages, indications, and methods of use for products referred to in this supplement are not necessarily the same as indicated in the package insert for the product and may reflect the clinical experience of the authors, presenters, and/or panelists or may be derived from the professional literature or other clinical sources. Consult complete prescribing information before administering. 
